\title{
TARMED-Paradox verlangt nach Notmassnahmen für die Psychiatrie
}

\author{
P. Hauser, U. Fischer, H. Lachenmeier \\ Ständige Tarifkommission der FMPP (Foederatio medicorum psychiatricorum et psychotherapeuticorum)
}

Erinnern Sie sich? Mit dem neuen Arzttarif soll die intellektuelle Arbeit gegenüber der technischen aufgewertet werden. Paradoxerweise erfährt ausgerechnet die psychiatrisch/psychotherapeutische Arbeit mit der Einführung des TARMED im IV/UV/MV-Bereich statt einer Aufwertung eine Abwertung. Diese skandalöse Tatsache verlangt nach sofortigen Notmassnahmen.

\section{Das Einkommen praktizierender Psychiater im Vergleich}

Die Einkommen der Psychiater und der Kinderund Jugendpsychiater betragen rund die Hälfte der durchschnittlichen Ärzteeinkommen. Wird der Vergleich nicht zum generellen Durchschnitt, sondern zwischen der Psychiatrie und allen anderen Fachbereichen gezogen, fällt das Resultat noch katastrophaler aus. Fast hat man sich daran gewöhnt.

Viele Kollegen anderer Fachrichtung gehen von der falschen Annahme aus, dass die niedrigen Psychiatrieeinkommen an kürzeren Arbeitszeiten liegen. Die ROKO-Zahlen belegen, dass die Arbeitszeit der Psychiater vergleichbar mit derjenigen der Anästhesiologen, Dermatologen, HNO-Ärzte, Augenärzte u. a. ist. Die Unterschiede im Durchschnittseinkommen dieser Fachgruppen betragen dabei bis zu Faktor drei. Es gibt weder fachliche noch betriebswirtschaftliche Gründe für diese Unterschiede.

\section{Die Situation der Psychiatrie im TARMED}

Im April 2001 anerkannte die Ärztekammer die Psychiatrie als eines der dringlich zu verbessernden Fachgebiete in den anstehenden RE-Verhandlungen zu TARMED. Die psychiatrischen Fachgebiete lieferten dazu mit aufwendigen Studien neue Daten zu Produktivität sowie Infrastrukturkosten. In der nun vorliegenden Einführungsversion $1.1 \mathrm{r}$ sind diese Daten noch immer nicht berücksichtigt.

Vor allem die Kinder- und Jugendpsychiater rechnen mit Einführung des TARMED im Bereiche der eidgenössischen Versicherungen ab 1. Mai 2003 einen wesentlichen Teil ihrer Arbeit mit dem neuen Tarif ab. Zwischen 30 und 70\% der Leistungen gehen als medizinische Eingliederungsmassnahmen bei psychischen Leiden, Geburtsgebrechen oder schweren Sprachgebrechen zu Lasten der Invalidenversicherung.

Die Einführung des TARMED ab Mai 2003 hat einen entscheidenden, negativen Einfluss insbesondere auf die Einkünfte der Kinder- und Jugendpsychiater.

\section{Parallelerhebung für die Psychiatrie}

Die Ständige Tarifkommission STK der beiden psychiatrischen Fachgesellschaften veranlasste eine repräsentative Erhebung in 13 kinder- und jugendpsychiatrischen Praxen in den Kantonen Zürich und Aargau. Die Erhebung fand in der letzten Märzwoche statt, um noch vor Einführung von TARMED konkrete Vergleichszahlen zu den Umsätzen zwischen altem IV-Tarif und TARMED in der Kinder- und Jugendpsychiatrie zu erhalten. Die Durchführung der Erhebung wurde freundlicherweise von der Ärztekasse übernommen.

Die teilnehmenden Ärztinnen und Ärzte rechneten ihre Leistungen anhand vorbereiteter Eingabeblätter der Ärztekasse je separat sowohl nach dem alten IV/MV/SUVA-Tarif als auch nach dem neuen TARMED ab. Die Erhebungsformulare wurden von der Ärztekasse zentral verarbeitet und ausgewertet.

Sämtliche erhobenen Daten konnten verwertet werden. Mit einem Volumen von rund Fr. 30 000.- aus allen 137 Fällen, die während der Erhebungswoche in den 13 ausgewählten Praxen mit einer oder mehreren Konsultationen behandelt wurden, ist die Erhebung genügend aussagekräftig, um gültige Vergleichszahlen zu liefern.

Der Gesamtumsatz der 13 Ärzte reduzierte sich selbst bei optimaler Anwendung des TARMED von Fr. 30023.13 nach altem IV-Tarif auf Fr. 29411.85 nach TARMED. Dies bedeutet eine Umsatzeinbusse von 2,04\%. Der Anteil an Praxisunkosten bleibt gleich. Er liegt laut ROKO [1] bei $49,3 \%$. Somit resultiert ein nachgewiesener Einkommensverlust von durchschnittlich $4 \%$ 
Tabelle 1

Parallelerhebung Kinder- und Jugendpsychiatrie März 2003; Eckdaten.

\begin{tabular}{ll} 
Erhebung durch & Ärztekasse Zürich \\
\hline Erhebungszeitraum & 24.-30. März 2003 \\
\hline Kantone & ZH und AG \\
\hline Teilnehmende Fachärzte/-ärztinnen & 13 \\
\hline Erhebungsumfang & $\begin{array}{l}\text { Sämtliche behandelten Fälle } \\
\text { des/r Facharztes/-ärztin in der Woche 9/03, } \\
\text { die über IV abgerechnet wurden }\end{array}$ \\
\hline Anzahl Fälle total & 137 \\
\hline Anzahl Fälle ausgewertet & 137 \\
\hline Fälle pro Facharzt/-ärztin & $8(1-25)$
\end{tabular}

2 FMH-Ärztestatistik 2001. Schweiz Ärztezeitung 2002; 83(18):871-91

3 Hasler N. Einkommensverhältnisse der freien Ärzteschaft der Schweiz. Schweiz Ärztezeitung 2002;83(46):2481-7 gegenüber dem Ausgangswert. Wird dieser Verlust in Relation zur anerkannt angestrebten Tarifverbesserung für die Psychiatrie gesetzt, so bedeutet dies einen Verlust von mindestens 15\%.

\section{Fazit}

Wir fragen nochmals: Wie um Himmels willen ist es $\mathrm{zu}$ rechtfertigen, dass die einkommensschwächsten Fachgruppen, die ausschliesslich intellektuell/emotionale Leistungen erbringen, im TARMED, der die intellektuell/emotionalen Leistungen aufbessern und die Einkommensunterschiede ausgleichen sollte, bereits mit dem ersten Einführungsschritt des neuen Tarifs nachweislich an Einkommen einbüssen?
Die Situation wird für die Praxispsychiatrie auch im KVG-Bereich ab 2004 nicht besser: das anerkannte Minimalziel von Fr 200.- Umsatz pro Stunde wird mit den zu erwartenden Taxpunktwerten nicht erreicht werden.

Die psychiatrische Versorgung der Schweiz wird in hohem Mass von der Praxispsychiatrie geleistet. Bereits heute besteht vielerorts eine Unterversorgung. Je nach Gegend und benötigter Behandlungsform bestehen Wartezeiten bis zu einem halben Jahr. Die Institutionen leiden seit Jahren an einem bedrohlichen Assistentenmangel. Die Dichte der Praxispsychiater wird somit weiter abnehmen. Gleichzeitig steigt der Bedarf an Behandlungen. Der Versorgungsnotstand Psychiatrie wird sich weiter verschärfen.

Ein wesentlicher Faktor, der zur Entwicklung dieses Notstandes geführt hat, ist die ungenügende Entlöhnung der psychiatrischen Arbeit. Ein junger Arzt benötigt übermässig hohen Idealismus, um sich im Wissen um diese Einkommensaussichten für eine Facharztausbildung in Psychiatrie/Psychotherapie zu entscheiden eine Ausbildung, von der (als Unikat unter den Facharztausbildungen) ein grosser Teil der obligatorischen Ausbildung in der Freizeit und auf eigene Kosten bewältigt werden muss.

Angesichts der drohenden weiteren Verschlechterung der psychiatrischen Einkommen, der bereits prekären Versorgungslage und des sich verschärfenden Nachwuchsmangels sind sofortige Notmassnahmen im TARMED unumgänglich.

Tabelle 2

Parallelerhebung Kinder- und Jugendpsychiatrie März 2003; Ergebnisse.

\begin{tabular}{|c|c|c|c|c|c|}
\hline Fallkosten & IV alt, in Fr. & $\%$ & IV TARMED & $\%$ & Differenz in \% \\
\hline Total & 30023.13 & & 29411.85 & & \\
\hline Fallkostendurchschnitt & 229.25 & & 226.02 & & \\
\hline Umsatz IV-Patienten in der Erhebungswoche (pro Facharzt/-ärztin) & 2309.47 & $100 \%$ & 2262.45 & 97,96 & $-2,04$ \\
\hline
\end{tabular}

